\title{
Critical involvement of RQCD1 in the EGFR-Akt pathway in mammary carcinogenesis
}

\author{
MASAHIKO AJIRO $^{1}$, TOSHIHIKO NISHIDATE ${ }^{1}$, TOYOMASA KATAGIRI ${ }^{1,2}$ and YUSUKE NAKAMURA $^{1}$ \\ ${ }^{1}$ Laboratory of Molecular Medicine, Human Genome Center, Institute of Medical Science, The University of Tokyo, Tokyo; \\ ${ }^{2}$ Division of Genome Medicine, Institute for Genome Research, The University of Tokushima, Tokushima, Japan
}

Received May 18, 2010; Accepted July 16, 2010

DOI: 10.3892/ijo_00000760

\begin{abstract}
We previously reported an important role of RQCD1 in mammary carcinogenesis through the interaction with Grb10 interacting GYF protein 1 (GIGYF1), Grb10 interacting GYF protein 2 (GIGYF2) and growth factor receptor binding protein 10 (Grb10). In this study, we investigated the biological mechanism of RQCD1 in regulation of the Akt activity as the downstream signal of epidermal growth factor receptor (EGFR). Knockdown of RQCD1 reduced the Akt phosphorylation level that was induced by epidermal growth factor (EGF) stimulation. We found a possible formation of the big complex involved in the Akt activity including Akt, EGFR, GIGYF1 and GIGYF2, Grb10 and RQCD1. We subsequently defined that a region corresponding to 620-665th amino acids of GIGYF1 and 667-712th amino acids of GIGYF2 interacted with RQCD1. Furthermore, we found that RQCD1 was required for enhancement of the interaction of Grb10 with GIGYF1 and GIGYF2. Our findings in this study imply the functional mechanism of RQCD1 in the Akt activity regulation as a mediator in the EGFR-signaling pathway.
\end{abstract}

\section{Introduction}

Breast cancer is one of the most prevalent types of cancer with 1,151,298 new cases and 410,712 deaths according to the global statistics in 2002 (1). Although molecular targeting drugs such as trastuzumab, lapatinib, aromatase inhibitors and tamoxifen exhibited clinical benefits in breast cancer treatment, these drugs cause severe adverse effects to some extent $(2,3)$. We extensively screened breast cancer cellspecific novel molecular targets, that can be applied for drug development with the minimum risk of adverse reactions,

Correspondence to: Dr Yusuke Nakamura, Laboratory of Molecular Medicine, Human Genome Center, Institute of Medical Science, The University of Tokyo, 4-6-1 Shirokanedai, Minato-ku, Tokyo 108-8639, Japan

E-mail: yusuke@ims.u-tokyo.ac.jp

Key words: RQCD1, Akt, EGFR, breast cancer through genome-wide cDNA microarray analysis $(4,5)$, and successfully identified such molecules and characterized their biological functions (6-16).

We have previously reported an important role of the required for cell differentiation 1 homologue (RQCD1) in mammary carcinogenesis and demonstrated that it was required for the maintenance of constitutive Akt activation in breast cancer cells (13). RQCD1 was originally isolated as the essential molecule for meiosis initiation in fission yeast Schizosaccharomyces pombe (17) and subsequently reported its requirement in several cell differentiation systems, such as endodermal-like differentiation and lung morphogenesis, suggesting its critical roles in the early embryonic and fatal stages (18-21). Functional relationship of RQCD1 to the breast cancer cell growth was demonstrated in our previous study (13). We found that RQCD1 interacted with Grb10 interacting GYF protein 1 (GIGYF1) and Grb10 interacting GYF protein 2 (GIGYF2), which were originally identified as interacting molecules with amino-terminal region of growth factor receptor binding protein 10 (Grb10), and were indicated to enhance the Akt activity (22). Grb10 has been identified as an interacting molecule for tyrosine-phosphorylated carboxy-terminus region of the epidermal growth factor receptor (EGFR) (23). Grb10 functions as an adaptor molecule for various receptor tyrosine kinases including EGFR, insulin-like growth factor-I receptor, insulin receptor, platelet-derived growth factor receptor, Met, c-Kit, Ret and ELK (24-27), and mediates activation of various cytoplasmic molecules such as Akt, phosphatidylinositol 3-kinase (PI3K) p85, Gab1, MEK and Raf-1 (27-30). In breast cancer cell lines, knockdown of RQCD1, GIGYF1, GIGYF2, or Grb10 significantly reduced the Akt activity and cell proliferation without affecting the Erk1/2 activity (13 and unpublished data), suggesting their significance in the Akt activity regulation in breast cancer cells. However, although significance of RQCD1 in the maintenance of constitutive Akt activity was indicated, detailed regulatory mechanism of constitutive Akt activation by RQCD1 overexpression in breast cancer cells was not fully elucidated.

In this study, we investigated possible roles of RQCD1 in linking epidermal growth factor (EGF) stimulation and the Akt activation using three EGF-responsive breast cancer cell lines. We demonstrate a complex formation of RQCD1 and EGFR-downstream molecules and its important role to stabilize the interaction of Grb10 with GIGYF1 and GIGYF2. These 
findings provide the functional mechanism of RQCD1 overexpression in the Akt activation in breast cancer cells.

\section{Materials and methods}

Cell lines. Human breast cancer cell lines MDA-MB-231, MCF-7, BT-549 and HCC1937, human cervical cancer cell line HeLa and HEK293T were purchased from American Type Culture Collection (ATCC; Rockville, MD, USA). Human breast cancer cell line HBC-5 was kindly provided by Dr Takao Yamori in Division of Molecular Pharmacology, Cancer Chemotherapy Center, Japanese Foundation for Cancer Research. MDA-MB-231 cells were cultured in Leibovitz's L-15 medium (Invitrogen, Carlsbad, CA, USA), supplemented with $10 \%$ fetal bovine serum (Cansera International, Ontario, Canada) and $1 \%$ antibiotic/antimycotic solution (Sigma-Aldrich, St. Louis, MO, USA), and maintained at $37^{\circ} \mathrm{C}$ in the atmosphere of humidified air without $\mathrm{CO}_{2}$. MCF-7, BT-549, HCC1937 and HBC-5 cells were cultured in RPMI-1640 medium (Invitrogen), supplemented with $10 \%$ fetal bovine serum (Cansera International) and $1 \%$ antibiotic/antimycotic solution (Sigma-Aldrich), and maintained at $37^{\circ} \mathrm{C}$ in the atmosphere of humidified air with $5 \% \mathrm{CO}_{2}$. HeLa and HEK293T cells were cultured in MEM (Invitrogen) and DMEM (Invitrogen), respectively, supplemented with $10 \%$ fetal bovine serum (Cansera International) and $1 \%$ antibiotic/antimycotic solution (Sigma-Aldrich), and maintained at $37^{\circ} \mathrm{C}$ in the atmosphere of humidified air with $5 \% \mathrm{CO}_{2}$.

Antibodies. Rabbit polyclonal antibodies for Akt (no. 9272), EGFR (no. 2232) and phosphorylated EGFR (Tyr1068) (no. 2234), and mouse monoclonal antibody against phosphorylated Akt (Ser473) (no. 4051) were purchased from Cell Signaling Technology (Danvers, MA, USA). Mouse monoclonal antibody against $B$-actin (Ac-15) and FLAG-tag (M2) were purchased from Sigma-Aldrich. Rabbit polyclonal antibody against influenza hemagglutinin (HA) tag (Y-11) and mouse monoclonal antibody against glutathione-Stransferase (GST) (B-14) were purchased from Santa Cruz Biotechnology (Santa Cruz, CA, USA). Rabbit polyclonal antibody against RQCD1 was previously developed in our laboratory (13). Horseradish peroxidase (HRP)-conjugated goat anti-rabbit IgG (sc-2004), anti-mouse IgG (sc-2005) and anti-rat IgG (sc-2006) were purchased from Santa Cruz Biotechnology.

Construction of expression vectors. Mammalian expression vectors for carboxyl-terminally FLAG-tagged Akt, EGFR cytoplasmic region (His676-Ala1210), GIGYF1, GIGYF2, p85 PI3K and RQCD1 were constructed with pCAGGSn3FC. Mammalian expression vectors for carboxyl-terminally HAtagged GIGYF1, GIGYF2 and Grb10 were constructed with pCAGGSnHC. Mammalian expression vectors for aminoterminally HA-tagged partial proteins of GIGYF1 (nos. 1, 2, 3, 4 and 5) and GIGYF2 (nos. 1, 2, 3, 4 and 5) were constructed with pCAGGSnH3F. Bacterial expression vector for amino-terminally GST-tagged RQCD1 was constructed with pGEX6P.1 (GE Healthcare). Coding sequences of each of these genes were amplified from complementary DNA of
HBC-5 by PCR with KOD-Plus DNA polymerase (Toyobo, Osaka, Japan). DNA sequences of each construct were confirmed by DNA sequencing by ABI3700 (Applied Biosystems, Foster, CA, USA). Primer sets used for cloning PCR are as follows: 5'-CATGAATTCATGAGCGACGTGGCTATTG-3' and 5'-CATCTCGAGGGCCGTGCCGCTGGCCGAG-3' for Akt, 5'-CATGCGGCCGCATGCACATCGTTCGGAAGCG CAC-3' and 5'-CATCTCGAGTGCTCCAATAAATTCAC TGC-3' for EGFR cytoplasmic region, 5'-CATGAATTCGA TGAATGCATCCCTGGAGAG-3' and 5'-ATGCTCGAGTA AGGCCACTCGGATGC-3' for Grb10, 5'-CATGAATTCAT GAGTGCTGAGGGGTACC-3' and 5'-CATGCGGCCGCG ATCGCCTCTGCTGTGCATA-3' for PI3K $p 85,5^{\prime}$-GTTAA GTAGCGGCCGCTCATGGCAGCAGAGACACTCAAC-3' and 5'-CCGCTCGAGTCATATGTCCCAAAGACTGGC-3' for GIGYF1 (no. 1), 5'-GTTAAGTAGCGGCCGCTCAT GGCAGCAGAGACACTCAAC-3' and 5'-CCGCTCGAG TCATTTGAGCGCCTGGAGCTGC-3' for GIGYF1 (no. 2), 5'-GTTAAGTAGCGGCCGCTCATGGCAGCAGAGAC ACTCAAC-3' and 5'-CCGCTCGAGTCAGACCAGCTGG AGAAACTG-3' for GIGYF1 (no. 3), 5'-GTTAAGTA GCGGCCGCTCATGGCAGCAGAGACACTCAAC-3' and 5'-ATGCTCGAGTCACTCCAGATCTCCGGGTGG -3' for GIGYF1 (no. 4), 5'-CATGCGGCCGCTGATGATGAAGGC TTGAAGC-3' and 5'-CCGCTCGAGGTAGTCATCCACGC TCTC-3' for GIGYF1 (no. 5), 5'-GTTAAGTAGCGGCCGC TCATGGCAGCGGAAACGCAGAC-3' and 5'-ATGCT CGAGTCAAAGATCCCATACACTACCAC-3' for GIGYF2 (no. 1), 5'-GTTAAGTAGCGGCCGCTCATGGCAGCGGA AACGCAGAC-3' and 5'-ATGCTCGAGTCAGTTCTGAT CAGATATTCTC-3' for GIGYF2 (no. 2), 5'-GTTAAGT AGCGGCCGCTCATGGCAGCGGAAACGCAGAC-3' and 5'-ATGCTCGAGTCATTGTTGTATTAAAAACTGCTGG-3' for GIGYF2 (no. 3), 5'-GTTAAGTAGCGGCCGCTCATGG CAGCGGAAACGCAGAC-3' and 5'-ATGCTCGAGTCAA TCAGGTTCTGTGGAAACAC-3' for GIGYF2 (no. 4), and 5'-CATGCGGCCGCTGATGAAGAAGGTCTCAAAC-3' and 5'-CCGCTCGAGGTAGTCATCCAACGTCTC-3' for GIGYF2 (no. 5), respectively. Underlining indicates recognition sites by restriction enzymes. Primer sets for cloning PCR of GIGYF1, GIGYF2 and RQCD1 were as described previously (13).

EGF stimulation experiment. For EGF stimulation analysis, $2.5 \times 10^{5}$ cells of HeLa and MDA-MB-231, or 4.0x10 cells of MCF-7 were seeded on a $10-\mathrm{cm}$ plate. Twenty-four hours after seeding, cells were transfected with siRNA targeting $R Q C D 1$ (target sequence: 5'-GATCTATCAGTGGATC AAT-3') or EGFP (target sequence: 5'-GCAGCACGACTTC TTCAAG-3') using Lipofectamine RNAiMAX (Invitrogen), as supplier's instructions. Forty-eight hours after the transfection of siRNA, cells were washed with PBS (-) and culture medium was substituted to serum-starved medium. After serum starvation for $24 \mathrm{~h}$, culture medium was substituted to medium containing $50 \mathrm{ng} / \mathrm{ml}$ of EGF without FBS. Cells were harvested 5 and $10 \mathrm{~min}$ after EGF treatment as well as before EGF addition ( $0 \mathrm{~min}$ ). For Akt activity analysis, cells were harvested with $200 \mu 1 / 10 \mathrm{~cm}$ plate of $2 \mathrm{X}$ Laemmli buffer containing $100 \mathrm{mM}$ Tris- $\mathrm{HCl}$ (pH 6.8), 2\% SDS, $16 \%$ glycerol, 4\% 2-mercaptoethanol, followed by sonication. 
Akt activity levels were subsequently assessed by densitometric analysis of Western blot results by Image J (31). For GST pull-down assay, cells were harvested with cell lysis buffer containing $50 \mathrm{mM}$ Tris- $\mathrm{HCl}$ ( $\mathrm{pH} 7.4$ ), $150 \mathrm{mM} \mathrm{NaCl}$, $1 \mathrm{mM}$ EDTA, $0.25 \%$ sodium deoxycholate, $0.5 \%$ NP-40, $1 \%$ Protease Inhibitor Cocktail Set II (Merck, Darmstadt, Germany) and $0.1 \%$ Protease Inhibitor Cocktail Set III (Merck), and applied for GST pull-down assay.

GST pull-down assay. HeLa cells treated with $50 \mathrm{ng} / \mathrm{ml}$ EGF for 0,5 and $10 \mathrm{~min}$ or HBC-5 cells cultured in serum-starved medium for $24 \mathrm{~h}$ were washed with ice-cold PBS (-), harvested with cell lysis buffer, and stroked 5 times with $0.6 \mathrm{~mm}$ gauge syringe. Cell lysates were then incubated on ice for $15 \mathrm{~min}$ and cleared by centrifugation at $14,000 \mathrm{rpm}$ for $15 \mathrm{~min}$ at $4^{\circ} \mathrm{C}$. Protein concentration of each cell lysate was quantified by Bio-Rad Protein Assay (Bio-Rad Laboratories, Hercules, CA, USA). For pre-cleaning, $1 \mathrm{mg}$ of cell lysate protein was incubated with $15 \mu \mathrm{l}$ of glutathione sepharose $4 \mathrm{~B}$ for $60 \mathrm{~min}$ at $4^{\circ} \mathrm{C}$, and the supernatant was then incubated with $20 \mu \mathrm{g}$ of GST-RQCD1 or equivalent molecular number of GST for $120 \mathrm{~min}$ at $4^{\circ} \mathrm{C}$, followed by incubation with $15 \mu \mathrm{l}$ of glutathione sepharose $4 \mathrm{~B}$ for $60 \mathrm{~min}$ at $4^{\circ} \mathrm{C}$. Beads were washed three times with $500 \mu 1$ of cell lysis buffer and once with $500 \mu \mathrm{l}$ of TBS [50 mM Tris- $\mathrm{HCl}(\mathrm{pH} 7.4), 150 \mathrm{mM}$ $\mathrm{NaCl}$, and $1 \mathrm{mM}$ EDTA]. Bead-bound proteins were eluted with $20 \mu 1$ of $2 \mathrm{X}$ Laemmli buffer and subsequently analyzed by Western blotting. For input controls, $10 \mu \mathrm{g}$ of cell lysate proteins were applied. For the analysis of exogenous proteins, expression vectors for FLAG-GIGYF1, FLAG-GIGYF2, HA-Grb10 and FLAG-PI3K p85 were transfected with FuGene6 (Roche, Basel, Switzerland) for $48 \mathrm{~h}$ prior to GST pull-down assay.

Preparation of recombinant proteins. Recombinant proteins of GST and GST-RQCD1 were prepared as described previously (13). For the preparation of recombinant proteins of FLAG-Akt, FLAG-EGFR cytoplasmic region, FLAGGIGYF1, Flag-GIGYF2 and HA-Grb10, HeLa cells were transfected with expression vectors for each protein. Cells were harvested with cell lysis buffer at $48 \mathrm{~h}$ after transfection and $15 \mathrm{mg}$ of cell lysate proteins were rotated with $50 \mu \mathrm{l}$ of sepharose 4B (Sigma-Aldrich) for $60 \mathrm{~min}$ at $4^{\circ} \mathrm{C}$ and supernatants were further rotated with $25 \mu 1$ of anti-HA or FLAG agarose (Sigma-Aldrich) for $60 \mathrm{~min}$ at $4^{\circ} \mathrm{C}$. Agarose beads were then washed 4 times with $5 \mathrm{ml}$ of cell lysis buffer and once with $5 \mathrm{ml}$ of TBS, and eluted by incubating with $500 \mu \mathrm{g} / \mathrm{ml}$ $3 \mathrm{X}$ Flag peptide or HA peptide for $60 \mathrm{~min}$ at $4^{\circ} \mathrm{C}$.

In vitro binding assay. In vitro binding assay was performed by incubating $0.5 \mu \mathrm{g}$ of each recombinant protein of FLAGAkt, FLAG-EGFR cytoplasmic region, HA-Grb10, FLAGGIGYF1 or FLAG-GIGYF2 with $1.0 \mu \mathrm{g}$ of GST-RQCD1 or equivalent molecular number of GST in $40 \mu \mathrm{l}$ of cell lysis buffer and rotated for $120 \mathrm{~min}$ at $4^{\circ} \mathrm{C}$. Each mixture of recombinant proteins was then transferred to microtubes containing $20 \mu \mathrm{l}$ of $50 \%$ slurry of glutathione sepharose $4 \mathrm{~B}$ (GE Healthcare) in cell lysis buffer and rotated for $60 \mathrm{~min}$ at $4^{\circ} \mathrm{C}$. Beads were washed twice with $500 \mu 1$ of cell lysis buffer and once with $500 \mu 1$ of TBS, followed by elution with
$10 \mu 1$ of $2 \mathrm{X}$ Laemmli buffer. Eluted samples were subsequently analyzed by Western blotting.

Co-immunoprecipitation assay. For co-immunoprecipitation assay, $1.0 \times 10^{6}$ cells of HEK293T were seeded to $10-\mathrm{cm}$ plate. Twenty-four hours after seeding, cells were transfected with respective combinations of expression vectors for HAtagged and FLAG-tagged proteins with FuGene6 (Roche) as per supplier's instructions. Thirty-six hours after co-transfection, cells were harvested with $500 \mu \mathrm{l}$ of cell lysis buffer followed by strokes with $0.6-\mathrm{mm}$ gauge syringe for 5 times and kept on ice for $15 \mathrm{~min}$. Cell lysate was then cleared with centrifugation at $14,000 \mathrm{rpm}$ at $4^{\circ} \mathrm{C}$ for $15 \mathrm{~min}$ and $1 \mathrm{mg}$ of cell lysate protein in the supernatant was incubated with $10 \mu 1$ of anti-Flag (M2) agarose (Sigma-Aldrich) or anti-HA (HA-7) agarose (Sigma-Aldrich) at $4^{\circ} \mathrm{C}$ for $1 \mathrm{~h}$. Agarose beads were washed three times with $500 \mu 1$ of cell lysis buffer and once with $500 \mu 1$ of TBS, followed by elution with $20 \mu 1$ of $2 \mathrm{X}$ Laemmli buffer. Input cell lysates and eluted samples were subsequently analyzed by Western blotting.

\section{Results}

RQCD1 is important in the Akt activation induced by EGFR stimulation in breast cancer cells. To evaluate the potential involvement of RQCD1 in the EGFR pathway, we first investigated knockdown effect of RQCD1 on the EGF-stimulated Akt activation that was evaluated by the phosphorylation level of Ser473 (32-34). Although Akt phosphorylation was at the basal level in HeLa, MCF-7 and MDA-MB-231 cells, we observed high levels of Ser473 phosphorylation immediately after the EGF stimulation. However, when we treated these cells with siRNA targeting RQCD1 for $72 \mathrm{~h}$ prior to the EGF stimulation, the Ser473 phosphorylation of Akt by the EGF stimulation was significantly diminished, concordant to significant suppression of the endogenous RQCD1 at the protein level (Fig. 1). On the other hand, reduction of the Akt activation was not observed when the cells were treated with control siRNA. Quantitative analysis of the Akt activity by densitometric analysis indicated 62,72 and $48 \%$ reduction of the Akt Ser473 phosphorylation level at $5 \mathrm{~min}$ after EGF treatment by RQCD1 knockdown in HeLa, MCF-7 and MDA-MB-231 cells, respectively. Reduction of the Akt phosphorylation level in RQCD1-knockdown cells was still evident at $10 \mathrm{~min}$ after EGF treatment in these three cell lines.

We subsequently evaluated the correlation between EGFR activity and RQCD1 in three breast cancer cell lines including HBC-5, in which the RQCD1-dependent constitutive Akt activation was observed (13). All of these cell lines expressed EGFR at a high level, and HBC-5 cells showed constitutive activation of EGFR which was confirmed by phosphorylation of Tyr1068, an EGFR autophosphorylation site induced by EGF (35) (Fig. 2A). In HBC-5 cells, knockdown of RQCD1 did not affect EGFR autophosphorylation, but the Akt phosphorylation (P-Akt) was significantly reduced (Fig. 2B).

RQCD1 formes a complex with EGFR downstream regulators. As reported previously (13), RQCD1 interacted with GIGYF1, GIGYF2 and Grb10. Since RQCD1 was likely to 
A
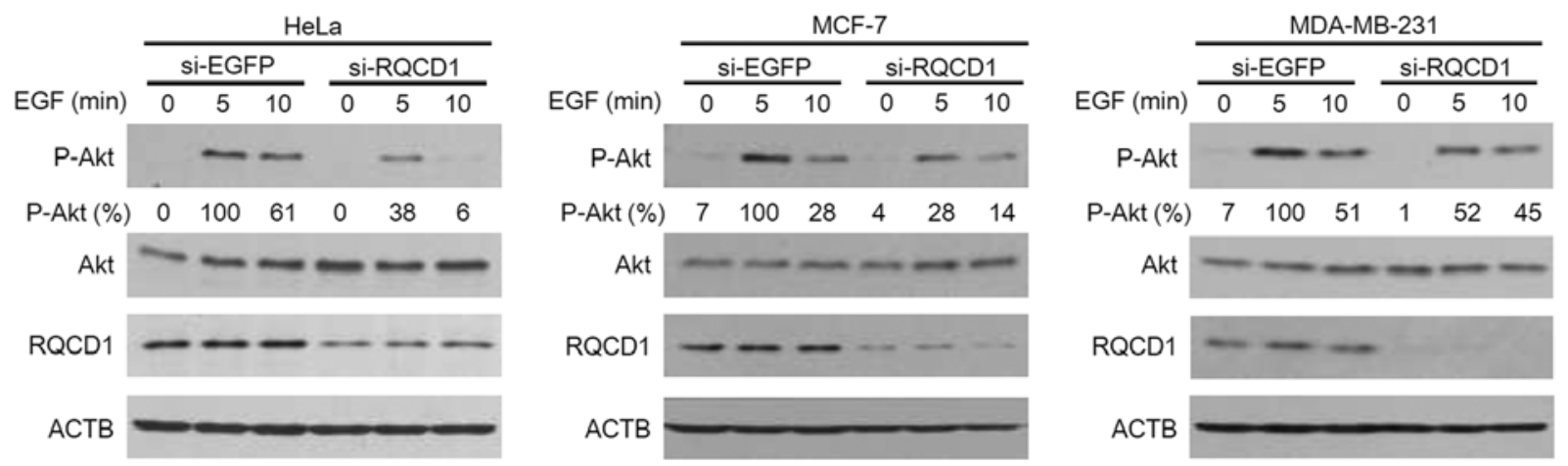

B
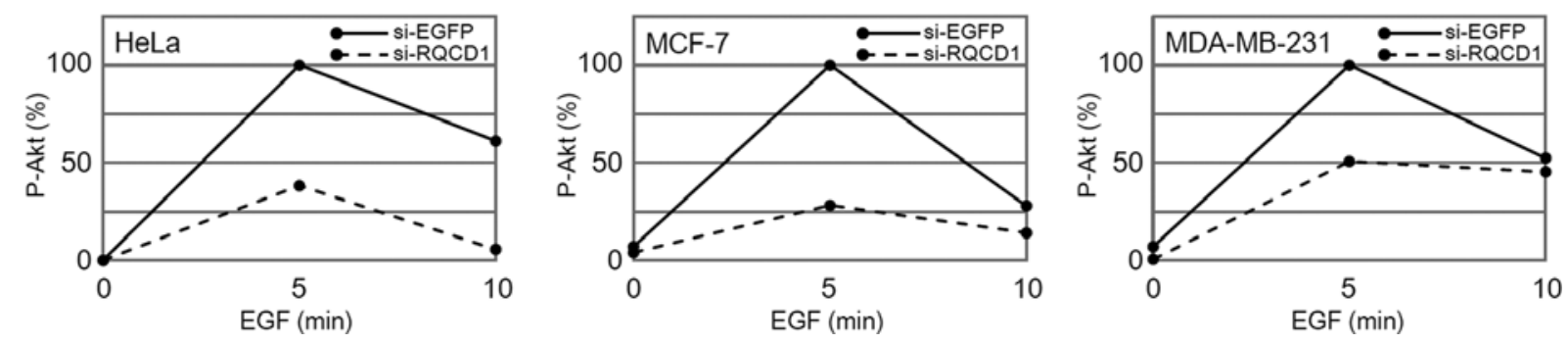

Figure 1. Knockdown effect of RQCD1 on the Akt activation induced by EGF stimulation. (A) HeLa, MCF-7 and MDA-MB-231 cells were treated with siRNA targeting RQCD1 or EGFR for $72 \mathrm{~h}$, and cultured in serum-starved medium for last $24 \mathrm{~h}$. Cells were then treated with $50 \mathrm{ng} / \mathrm{ml}$ EGF for indicated times and applied to Western blot analysis to examine phosphorylation of Akt on Ser473 (P-Akt), Akt, RQCD1 and ß-actin (ACTB). Relative Akt activation level (\%) was estimated by the intensity ratio of P-Akt/Akt compared with $5 \mathrm{~min}$ in siEGFP-transfected cells. (B) Relative Akt activation level is indicated in line graph. Vertical axis indicates relative intensity (\%) of P-Akt/Akt compared with 5 min in siEGFP-transfected cells. Horizontal axis indicates time after the EGF treatment.

be regulated by EGFR and interacted with Grb10, we hypothesized that RQCD1 might have the function as an adaptor molecule in the EGFR-signaling pathway. To test this possibility, we examined interaction of RQCD1 with EGFR as well as its reported downstream molecules, including Akt, GIGYF1, GIGYF2, Grb10 and p85 PI3K $(22,23,25,27)$. In HeLa cells, when we pulled down the interacting proteins with GST-RQCD1, Akt, EGFR, GIGYF1, GIGYF2 and Grb10 were co-immunoprecipitated with RQCD1, but p85 PI3K was not (Fig. 3A). Similar results were observed when we used HBC-5 cells, in which EGFR was constitutively activated (Fig. 3B).

Interaction analysis of RQCD1 by in vitro binding assay. Although GST pull-down assay using HeLa and HBC-5 cells indicated the potential complex formation of RQCD1 with various molecules related to EGF-Akt signaling pathway, the direct partner of the binding among the complex was unclear. Thus, we examined in vitro binding assay with purified recombinant proteins. Recombinant proteins of GST-RQCD1, FLAG-EGFR cytoplasmic region (His676-Ala1210), FLAGAkt, FLAG-GIGYF1, FLAG-GIGYF2 and HA-Grb10 were affinity-purified with glutathione-, anti-FLAG-, or anti-HAagarose resin. Their purity and specificity were confirmed by CBB staining and Western blotting for FLAG-, HA-, or GSTtag (Fig. 4A). Then, we performed binding assay using these purified recombinant proteins with RQCD1 and subsequent Western blot analysis for respective epitope-tags revealed specific interaction of each purified protein with RQCD1 in vitro, suggesting RQCD1 to be a core protein for constituting this complex (Fig. 4B).

Interacting region analysis of GIGYF1 and GIGYF2 with $R Q C D 1$. We further investigated interacting regions of GIGYF1 and GIGYF2 with RQCD1. First, expression vectors for amino-terminally HA-tagged full-length or partial proteins (nos. 1, 2, 3, 4 and 5) of GIGYF1 (Fig. 5A, upper panel) were co-transfected with FLAG-tagged RQCD1 in HEK293T cells. Subsequent immunoprecipitation for FLAGtag revealed interactions of full-length, nos. 1 and 5 GIGYF1 with RQCD1, suggesting 620-665th amino acids, commonly deleted region in nos. 2, 3 and 4 was potential interacting region between RQCD1 and GIGYF1 (Fig. 5B). Similarly we investigated an interacting region of GIGYF2 using expression vectors for amino-terminally HA-tagged full-length and partial proteins (nos. 1, 2, 3, 4 and 5) of GIGYF2, that were deleted for homologous regions corresponding to respective GIGYF1 partial proteins (Fig. 5A, lower panel) in HEK293T cells. Then, interactions of full-length and partial proteins of GIGYF2 with FLAG-RQCD1 were analyzed by co-immunoprecipitation assay. As a result, the interaction pattern of GIGYF2 partial proteins with RQCD1 was similar to that of GIGYF1, and a region corresponding to 667-712th amino acids of GIGYF2, that was homologous to 620-665th amino acids of GIGYF1, was the potential interacting region with RQCD1 (Fig. 5C). 
A

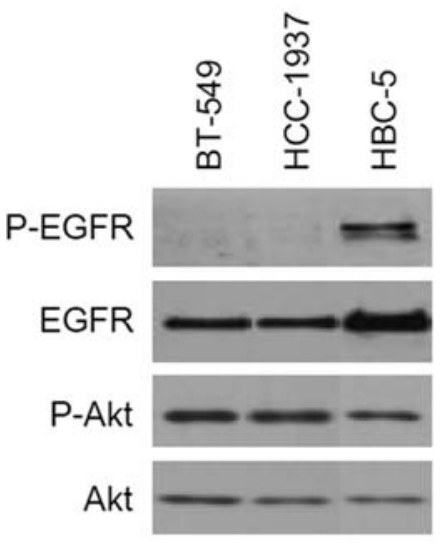

B

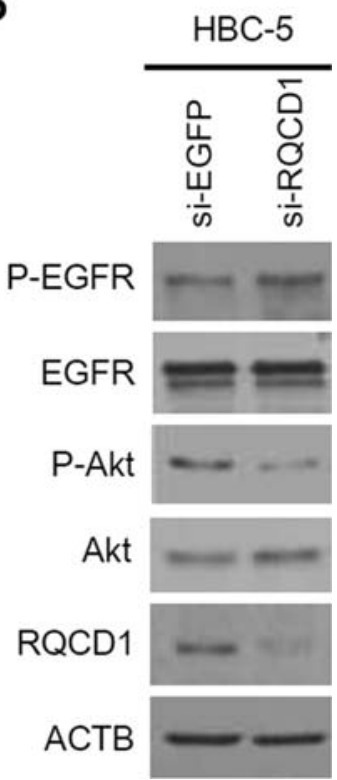

Figure 2. Constitutive EGFR activation status in breast cancer cell lines. (A) BT-549, HCC-1937 and HBC-5 cells were cultured in serum-starved medium for $24 \mathrm{~h}$. Cells were then analyzed by Western blotting for phosphorylated EGFR (Tyr1068) (P-EGFR), EGFR, P-Akt and Akt. (B) HBC-5 cells were treated with siRNA targeting RQCD1 or EGFP for $72 \mathrm{~h}$, and cultured in serum-starved medium for $24 \mathrm{~h}$, followed by Western blotting analysis for P-EGFR, EGFR, P-Akt, Akt, RQCD1 and ACTB.

A

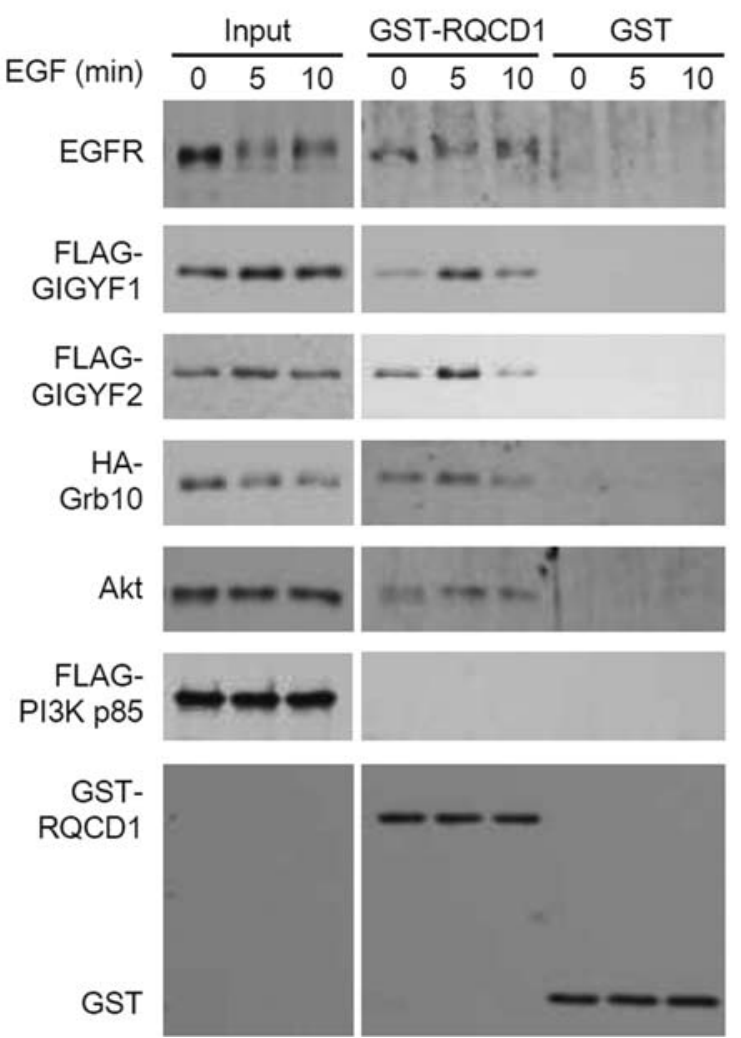

B

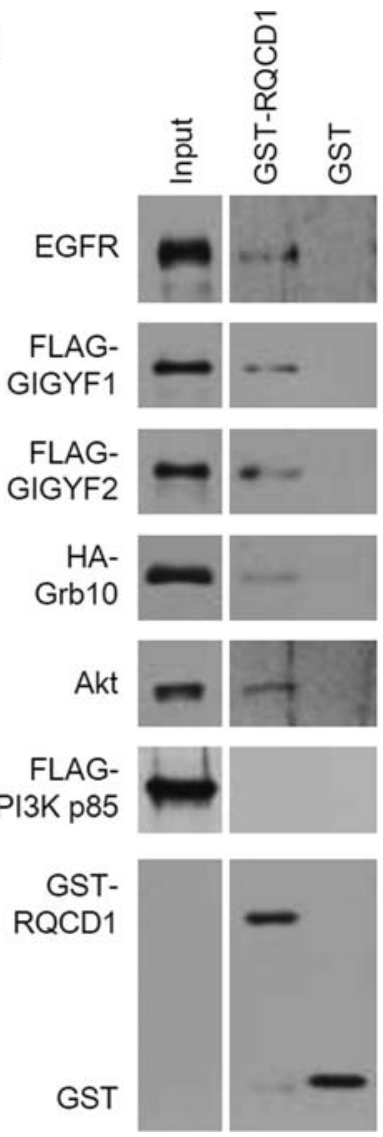

Figure 3. Interaction analysis of RQCD1 by GST pull-down assay. (A) HeLa cells were treated with $50 \mathrm{ng} / \mathrm{ml}$ of EGF for indicated times followed by GST pull-down with GST-RQCD1 or GST as a control. Pull-down products were then analyzed by Western blotting for EGFR, Akt, FLAG-tag (for FLAGGIGYF1, FLAG-GIGYF2 and FLAG-PI3K p85), HA (HA-Grb10), or GST (GST-RQCD1 and GST). For the investigation of FLAG-GIGYF1, FLAGGIGYF2, FLAG-PI3K p85 and HA-Grb10, each expression vector was transfected for $48 \mathrm{~h}$ prior to EGF treatment. A representative result of two independent experiments for each protein is indicated. (B) HBC-5 cells were cultured in serum-starved medium for 24 h followed by GST pull-down with GST-RQCD1 or GST as a control. Pull-down products were then analyzed by Western blotting. A representative result of two independent experiments for each protein is shown 
A

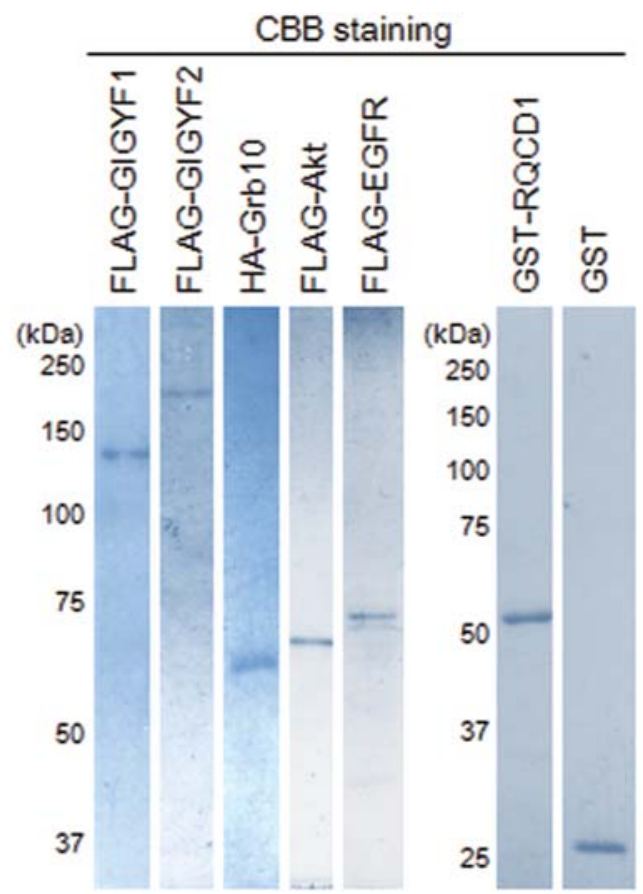

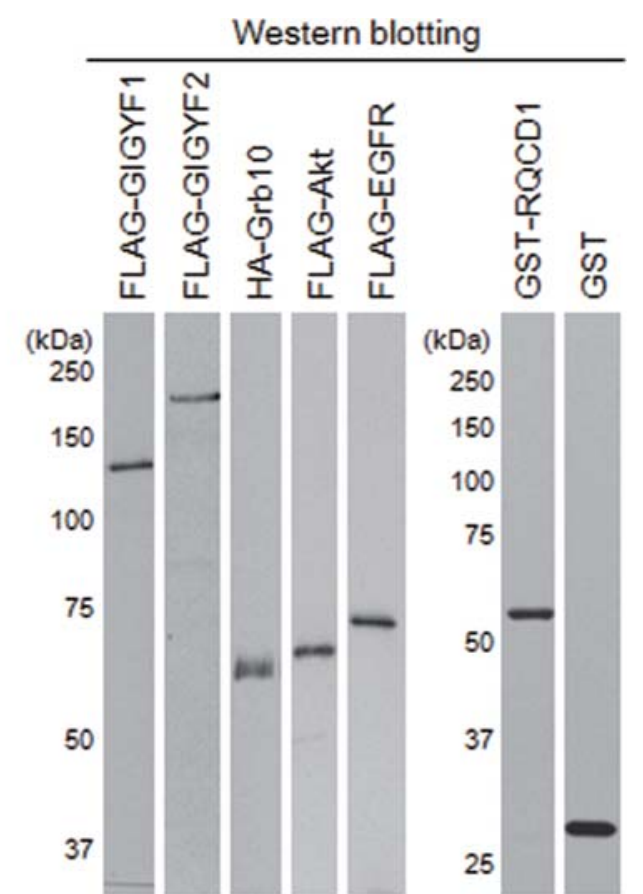

B

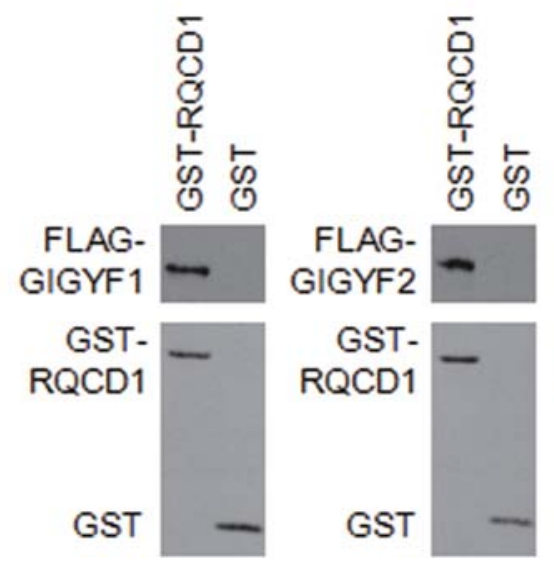

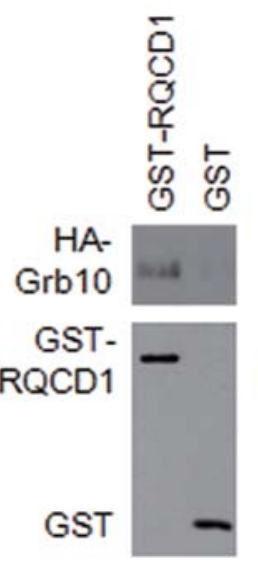

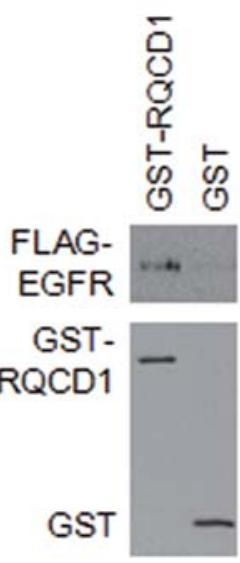

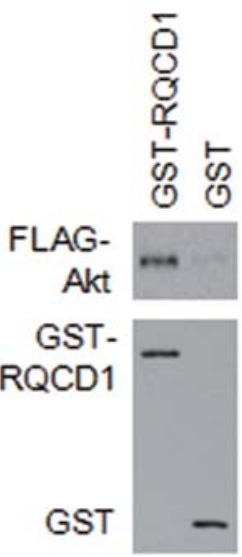

Figure 4. Interaction analysis of RQCD1 by in vitro binding assay. (A) After the affinity purification of FLAG-GIGYF1, FLAG-GIGYF2, HA-Grb10, FLAGAkt, FLAG-EGFR cytoplasmic region (His676-Ala1210), GST-RQCD1 and GST proteins, their purity and specificity were examined by CBB staining after SDS-PAGE (left panel) and Western blotting for each epitope tag (right panel), respectively. (B) Each purified protein was incubated with GST-RQCD1 or GST, and the pull-down assay was performed with glutathione sepharose 4B. Interaction of each recombinant protein was then evaluated by Western blotting for each epitope tag.

Significance of RQCD1 in the interaction of Grb10 with GIGYF1 and GIGYF2. To further investigate the role of RQCD1 in the complex formation, we examined knockdown effect of RQCD1 on the interactions between FLAG-GIGYF1 and HA-Grb10, and FLAG-GIGYF2 and HA-Grb10. Immunoprecipitation was performed using FLAG-tag antibody at $36 \mathrm{~h}$ after the transfection of expression vectors and $72 \mathrm{~h}$ after the transfection of siRNAs in HEK293T cells. As a result, we found significant reduction in the affinity in both FLAG-GIGYF1 and HA-Grb10, and FLAG-GIGYF2 and HA-Grb10 in RQCD1-knocked down cells compared with cells treated with control siRNA for EGFP (Fig. 6), suggesting the important role of RQCD1 in the stabilization of these interactions as the adaptor.

\section{Discussion}

In this study, we examined the function of RQCD1 in the signal transduction from EGFR to Akt activation. We observed reduction of EGF-induced Akt activation by the knockdown of RQCD1 in two EGF-responsive breast cancer cell lines MCF-7 and MDA-MB-231, as well as cervical cancer cell line HeLa, indicating RQCD1 was required for Akt activation as one of key components in the EGFRsignaling pathway (Fig. 1). We also examined possible roles of RQCD1 in the EGFR downstream activity using breast cancer cells in which Akt was constitutively activated. As a result, constitutive Akt activation in HBC-5 cells was found to be dependent on the constitutive EGFR activity as well as 
A
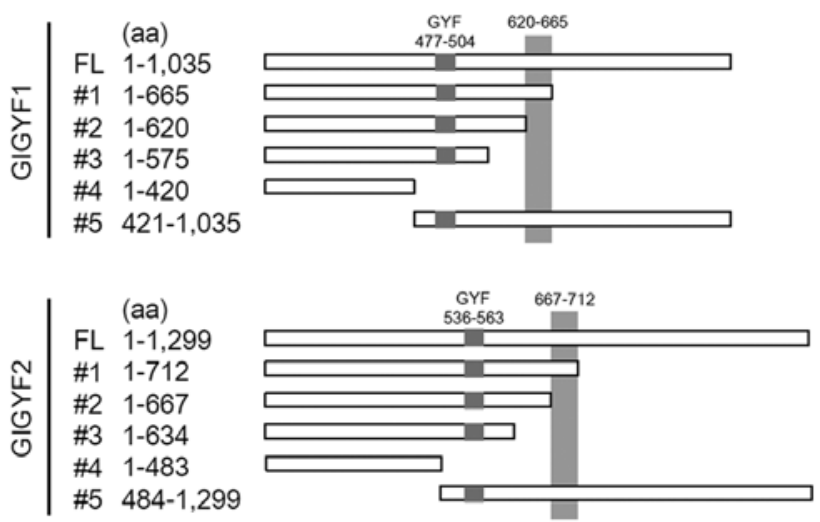

B

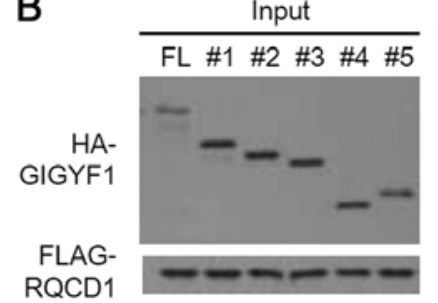

C

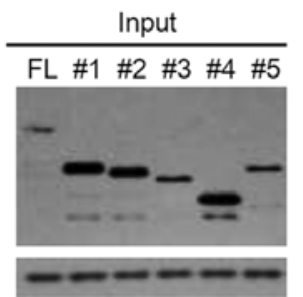

IP: FLAG

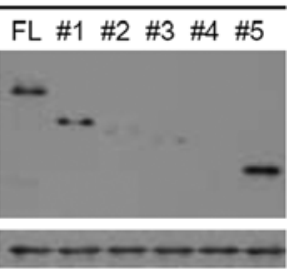

IP: FLAG

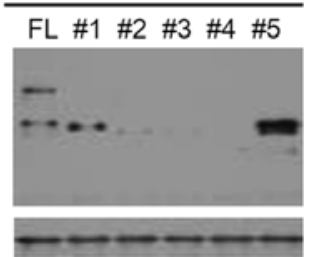

Figure 5. Interacting region analysis of RQCD1 with GIGYF1 and GIGYF2. (A) Structure of full-length (FL) and partial proteins (nos. 1, 2, 3, 4 and 5) of GIGYF1 (upper) and GIGYF2 (lower). (B and C) Immunoprecipitation for FLAG-tag was performed $36 \mathrm{~h}$ after co-transfection of FLAG-RQCD1 and aminoterminally HA-tagged full length (FL) or partial proteins (nos. 1, 2, 3, 4 and 5) of GIGYF1 (B) or GIGYF2 (C) in HEK293T. Western blotting was subsequently performed for HA-tag and FLAG-tag antibodies.

A

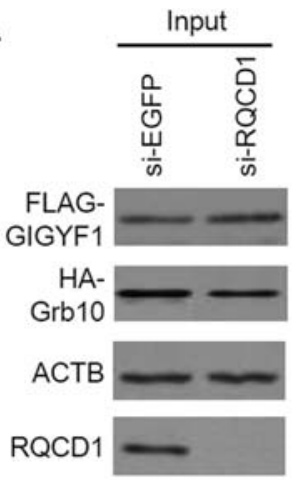

B

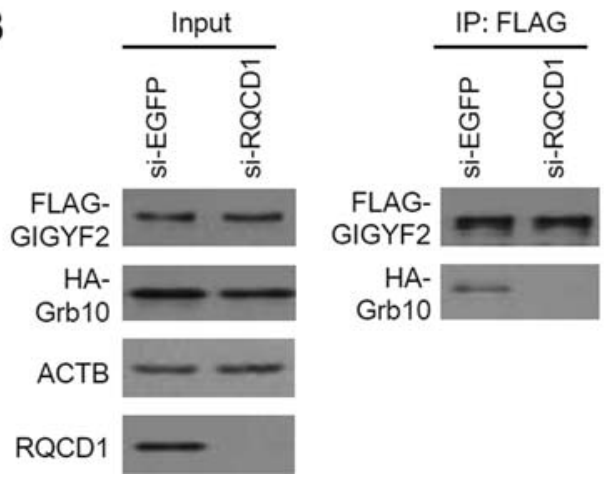

Figure 6. Knockdown effect of RQCD1 on the interaction of Grb10 with GIGYF1 and GIGYF2. (A and B) HEK293T cells were treated with siRNA targeting EGFP or $R Q C D 1$ for $36 \mathrm{~h}$, and transfected with expression vectors for FLAG-GIGYF1 and HA-Grb10 (A), or FLAG-GIGYF2 and HA-Grb10 (B), for additional $36 \mathrm{~h}$. Subsequently, immunoprecipitation using FLAGtag antibody was performed. Total cell lysates and immunoprecipitation products were analyzed by Western blotting. In (A) and (B), representative results of three independent experiments are demonstrated.

RQCD1 (Fig. 2). On the other hand, in BT-549 and HCC1937 cells, in which EGFR was not constitutively activated
(Fig. 2A), we found that RQCD1 overexpression played a key role in the Akt activation (13). Although EGFR was not constitutively activated in these cell lines, it might be possible that some other Grb10-interacting receptor tyrosine kinase is constitutively activated and its downstream activity is also regulated by RQCD1. The Akt activation mechanism in these cell lines should be investigated to further elucidate the function of RQCD1.

Interaction analysis of RQCD1 by GST pull-down assay and subsequent in vitro binding assay with purified proteins indicated potentially direct interaction of RQCD1 with EGFR, Akt, Grb10, GIGYF1 and GIGYF2 (Figs. 3 and 4). Although PI3K p85 was reported to interact with Grb10 leading to PI3K and Akt activations downstream of insulin receptor in Rat-1 embryonic fibroblast (29), our analysis did not show the interaction of PI3K p85 with the RQCD1 complex although interaction of RQCD1 with Grb10 was observed (Fig. 3). Another report also indicated that Grb10 was not involved in regulation of the PI3K activity (27). Thus, it was suggested that function of Grb10 to regulate PI3K activity could be different depending on the cell types and/or the upstream growth factor receptors. Our data imply that in the EGF-stimulated HeLa cells and in constitutively EGFR-activated HBC-5 cells, Grb10 and RQCD1 might not interact and regulate the PI3K activity (Fig. 3). On the other hand, Akt, EGFR and Grb10 showed constitutive interaction with RQCD1 upon EGF treatment. Constitutive interaction during growth factor stimulation was also reported for the interaction between Grb10 and Akt in COS-1, Mo7e and K562 cells, and Grb10 was indicated to recruit Akt to cellular membrane to mediate Akt activation (27). Considering similar constitutive interaction of RQCD1 with Grb10 and Akt, RQCD1 would also function in the recruitment process of Akt to EGFR, although the association and dissociation mechanism of RQCD1 remains to be elucidated. In response to EGF stimulation, GIGYF1 and GIGYF2 showed transient argumentation of their interactions with RQCD1 (Fig. 3A), 
suggesting these interactions were affected by such EGF stimulation-responsive events as phosphorylation or conformational changes in EGFR downstream molecules, although further investigation is required to fully understand the regulatory mechanism of the RQCD1 complex formation.

Through interaction analysis of RQCD1 with full-length or partial proteins of GIGYF1 and GIGYF2, segments corresponding to 620-665th and 667-712th amino acids were identified as potential interacting regions on GIGYF1 and GIGYF2, respectively, with RQCD1 (Fig. 5). Because these interacting regions were homologous to each other, it was suggested that GIGYF1 and GIGYF2 interact with RQCD1 competitively. However, GIGYF1 and GIGYF2 are likely to regulate Akt activation in a different way rather than redundantly because knockdown of either GIGYF1 or GIGYF2 decreased Akt activity (13). It might be possible that the binding of GIGYF1 and GIGYF2 confers a different interaction property to RQCD1, regulating a dynamic alteration of complex components during signal transduction.

Overexpression of EGFR is one of clinical features of breast cancer correlating with poor prognosis (36-40). Many types of molecular targeting drugs against EGFR have been developed for various cancer types including breast cancer (41-45). However, despite their clinical benefits, ubiquitous expression and physiological requirement of EGFR in noncancerous tissues led to various adverse effects such as severe skin toxicities, resulting in decreased quality of life of patients and interruption or discontinuation of anti-EGFR treatment $(46,47)$. However, these problems should be avoided if drugs are designed against cancer cell-specific EGFR downstream regulators instead of EGFR itself. Considering its importance in the downstream activation of EGFR, breast cancer cell proliferation, and specific expression pattern in breast cancer cells, RQCD1 could be an appropriate molecular target against EGFR pathway in a breast cancer cell-specific manner. Thus, although further functional mechanism of RQCD1 ought to be elucidated, findings in this study indicate the importance of RQCD1 in EGFR downstream activation, providing the possibility to interfere with oncogenic EGFR pathway specifically in breast cancer cells.

\section{Acknowledgements}

We appreciate Dr Chikako Fukukawa for helpful discussions, and Ms. Kie Naito, Ms. Kyoko Kijima and Ms. Yoshiko Fujisawa for their technical supports.

\section{References}

1. Parkin DM, Bray F and Ferlay J: Global cancer statistics, 2002. CA Cancer J Clin 55: 74-108, 2005.

2. Veronesi U, Boyle P, Goldhirsch A, Orecchia R and Viale G: Breast cancer. Lancet 365: 1727-1741, 2005.

3. Bange J, Zwick E and Ullrich A: Molecular targets for breast cancer therapy and prevention. Nat Med 7: 548-552, 2001.

4. Nishidate T, Katagiri T, Lin ML, et al: Genome-wide geneexpression profiles of breast-cancer cells purified with laser microbeam microdissection: identification of genes associated with progression and metastasis. Int J Oncol 25: 797-819, 2004.

5. Saito-Hisaminato A, Katagiri T, Kakiuchi S, Nakamura T, Tsunoda T and Nakamura Y: Genome-wide profiling of gene expression in 29 normal human tissues with a cDNA microarray. DNA Res 9: 35-45, 2002.
6. Park JH, Lin ML, Nishidate T, Nakamura Y and Katagiri T: PDZ-binding kinase/T-LAK cell-originated protein kinase, a putative cancer/testis antigen with an oncogenic activity in breast cancer. Cancer Res 66: 9186-9195, 2006.

7. Lin ML, Park JH, Nishidate T, Nakamura Y and Katagiri T: Involvement of maternal embryonic leucine zipper kinase (MELK) in mammary carcinogenesis through interaction with Bcl-G, a pro-apoptotic member of the Bcl-2 family. Breast Cancer Res 9: R17, 2007.

8. Shimo A, Nishidate T, Ohta T, Fukuda M, Nakamura Y and Katagiri T: Elevated expression of protein regulator of cytokinesis 1 , involved in the growth of breast cancer cells. Cancer Sci 98: 174-181, 2007.

9. Shimo A, Tanikawa C, Nishidate T, et al: Involvement of kinesin family member $2 \mathrm{C} /$ mitotic centromere-associated kinesin overexpression in mammary carcinogenesis. Cancer Sci 99: 62-70, 2008.

10. Ueki T, Nishidate T, Park JH, et al: Involvement of elevated expression of multiple cell-cycle regulator, DTL/RAMP (denticleless/RA-regulated nuclear matrix associated protein), in the growth of breast cancer cells. Oncogene 27: 5672-5683, 2008.

11. Lin ML, Fukukawa C, Park JH, et al: Involvement of overexpression of G-patch domain containing 2 in breast carcinogenesis. Cancer Sci 100: 1443-1450, 2009.

12. Kim JW, Akiyama M, Park JH, et al: Activation of an estrogen/estrogen receptor signaling by BIG3 through its inhibitory effect on nuclear transport of PHB2/REA in breast cancer. Cancer Sci 100: 1468-1478, 2009.

13. Ajiro M, Katagiri T, Ueda K, et al: Involvement of RQCD1 overexpression, a novel cancer-testis antigen, in the Akt pathway in breast cancer cells. Int J Oncol 35: 673-681, 2009.

14. Ueki T, Park JH, Nishidate T, Kijima K, Hirata K, Nakamura Y and Katagiri T: Ubiquitination and downregulation of BRCA1 by ubiquitin-conjugating enzyme E2T overexpression in human breast cancer cells. Cancer Res 69: 8752-8760, 2009.

15. Park JH, Nishidate T, Nakamura Y and Katagiri T: Critical roles of T-LAK cell-originated protein kinase in cytokinesis. Cancer Sci 101: 403-411, 2010.

16. Park JH, Nishidate T, Kijima K, et al: Critical roles of mucin 1 glycosylation by transactivated polypeptide $\mathrm{N}$-acetylgalactosaminyltransferase 6 in mammary carcinogenesis. Cancer Res 70: 2759-2769, 2010

17. Okazaki N, Okazaki K, Watanabe Y, Kato-Hayashi M, Yamamoto $\mathrm{M}$ and Okayama $\mathrm{H}$ : Novel factor highly conserved among eukaryotes controls sexual development in fission yeast. Mol Cell Biol 18: 887-895, 1998.

18. Gregory RC, Lord KA, Panek LB, Gaines P, Dillon SB and Wojchowski DM: Subtraction cloning and initial characterization of novel Epo-immediate response genes. Cytokine 12: $845-857,2000$

19. Hiroi N, Ito T, Yamamoto H, Ochiya T, Jinno S and Okayama H: Mammalian Rcd1 is a novel transcriptional cofactor that mediated retinoic acid-induced cell differentiation. EMBO J 21: 5235-5244, 2002 .

20. Haas M, Siegert M, Shurmann A, Sodeik B and Wolfes H: c-Myb protein interacts with Rcd-1, a component of the CCR4 transcription mediator complex. Biochemistry 43: 8152-8159, 2004.

21. Garapaty S, Mahajan MA and Samuels HH: Components of the CCR4-NOT complex function as nuclear hormone receptor coactivators via association with the NRC-interacting factor NIF-1. J Biol Chem 283: 6806-6816, 2008.

22. Giovannone B, Lee E, Laviola L, Giorgino F, Cleveland KA and Smith RJ: Two novel proteins that are linked to insulin-like growth factor (IGF-1) receptors by the Grb10 adapter and modulate IGF-1 signaling. J Biol Chem 34: 31564-31573, 2003.

23. Ooi J, Yajnik V, Immanuel D, Gordon M, Moskow JJ, Buchberg AM and Margolis B: The cloning of Grb10 reveals a new family of SH2 domain proteins. Oncogene 10: 1621-1630, 1995.

24. Stein E, Cerretti DP and Daniel TO: Ligand activation of ELK receptor tyrosine kinase promotes its association with Grb10 and Grb2 in vescular endothelial cells. J Biol Chem 271: 23588-23593, 1996.

25. He Weimin, Rose DW, Olefsky JM and Gustafson TA: Grb10 interacts differentially with the insulin receptor, insulin-like growth factor I receptor, and epidermal growth factor receptor via the Grb10 Src homology 2 (SH2) domain and a second novel domain located between the Pleckstrin homology and SH2 domains. J Biol Chem 273: 6860-6867, 1998. 
26. Wang J, Dai H, Yousaf N, et al: Grb10, a positive, stimulatory signaling adapter in platelet-derived growth factor BB-, insulinlike growth factor I-, and insulin-mediated mitogenesis. Mol Cell Biol 19: 6217-6228, 1999.

27. Jahn T, Seipel P, Urschel S, Peschel C and Duyster J: Role for the adaptor protein Grb10 in the activation of Akt. Mol Cell Biol 22: 979-991, 2002.

28. Nantel A, Huber M and Thomas DY: Localization of endogenous Grb10 to the mitochondria and its interaction with the mitochondrial-associated Raf1 pool. J Biol Chem 274: 35719-35724, 1999.

29. Deng Y, Bhattacharya S, Swamy OR, Tandon R, Wang Y, Janda $\mathrm{R}$ and Riedel $\mathrm{H}$ : Growth factor receptor-binding protein 10 (Grb10) as a partner of phosphatidylinositol 3-kinase in metabolic insulin action. J Biol Chem 278: 39311-39322, 2003.

30. Deng Y, Zhang M and Riedel H: Mitogenic roles of Gab1 and Grb10 as direct cellular partners in the regulation of MAP kinase signaling. J Cell Biochem 105: 1172-1182, 2008.

31. Abramoff MD, Magelhaes PJ and Ram SJ: Image processing with Image J. Biophotonics Int 11: 36-42, 2004.

32. Alessi DR, Andjelkovic M, Caudwell B, Cron P, Morrice N, Cohen P and Hemmings BA: Mechanism of activation of protein kinase B by insulin and IGF-1. EMBO J 15: 6541-6551, 1996.

33. Yang J, Cron P, Thompson V, Good VM, Hess D, Hemmings BA and Barford D: Molecular mechanism for the regulation of protein kinase B/Akt by hydrophobic motif phosphorylation. Mol Cell 9: 1227-1240, 2002.

34. Scheid MP, Marignani PA and Woodgett JR: Multiple phosphoinositide 3-kinase-dependent steps in activation of protein kinase B. Mol Cell Biol 22: 6247-6260, 2002.

35. Rojas M, Yao SY and Lin YZ: Controlling epidermal growth factor (EGF)-stimulated Ras activation in intact cells by a cell permeable peptide mimicking phosphorylated EGF receptor. J Biol Chem 271: 27456-27461, 1996.

36. Aziz SA, Pervez S, Khan S, Kayani N and Rahbar MH: Epidermal growth factor receptor (EGFR) as a prognostic marker: an immunohistochemical study on 315 consecutive breast carcinoma patients. J Pak Med Assoc 52: 104-110, 2002.
37. Buchholz TA, Tu X, Ang KK, et al: Epidermal growth factor receptor expression correlates with poor survival in patients who have breast carcinoma treated with doxorubicin-based neoadjuvant chemotherapy. Cancer 104: 676-681, 2005.

38. DiGiovanna MP, Stern DF, Edgerton SM, et al: Relationship of epidermal growth factor receptor expression to ErbB-2 signaling activity and prognosis in breast cancer patients. J Clin Oncol 23: 1152-1160, 2005.

39. Tzaida O, Gogas H, Dafni U, et al: Evaluation of the prognostic and predictive value of HER-1/EGFR in breast cancer patients participating in a randomized study with dose-dense sequential adjuvant chemotherapy. Oncology 72: 388-396, 2007.

40. Nieto Y, Nawaz F, Jones RB, et al: Prognostic significance of overexpression and phosphorylation of epidermal growth factor receptor (EGFR) and the presence of truncated EGFRvIII in locoregionally advanced breast cancer. J Clin Oncol 25: 4405-4413, 2007.

41. Hynes NE and Lane HA: ERBB receptors and cancer: the complexity of targeted inhibitors. Nat Rev Cancer 5: 341-354, 2005.

42. Tan AR, Yang X, Hewitt SM, et al: Evaluation of biologic end points and pharmacokinetics in patients with metastatic breast cancer after treatment with erlotinib, an epidermal growth factor receptor tyrosine kinase inhibitor. J Clin Oncol 22: 3081-3090, 2004.

43. Baselga J, Albanell J, Ruiz A, et al: Phase II and tumor pharmacodynamic study of gefitinib in patients with advanced breast cancer. J Clin Oncol 23: 5323-5333, 2005.

44. Geyer CE, Forster J, Lindquist D, et al: Lapatinib plus capecitabine for HER2-positive advanced breast cancer. N Engl J Med 355: 2733-2743, 2006.

45. Burstein HJ, Sun Y, Dirix LY, et al: Neratinib, an irreversible ErbB receptor tyrosine kinase inhibitor, in patients with advanced ErbB2-positive breast cancer. J Clin Oncol 28: 1301-1307, 2010.

46. Perez-Soler R and Saltz L: Cutaneous adverse effects with HER1/EGFR-targeted agents: Is there a silver lining? J Clin Oncol 23: 5235-5246, 2005.

47. Lacouture ME: Mechanisms of cutaneous toxicities to EGFR inhibitors. Nat Rev Cancer 6: 803-812, 2006. 